\title{
ALMOST EVERYWHERE CONVERGENCE OF LACUNARY TRIGONOMETRIC SERIES WITH RESPECT TO RIESZ PRODUCTS
}

\author{
J. PEYRIÈRE \\ (Received 17 October 1988) \\ Communicated by W. Moran
}

\begin{abstract}
Let $\left\{\lambda_{j}\right\}_{j \geq 0}$ be a sequence of positive integers such that $\lambda_{j+1} / \lambda_{j} \geq 3$ and $\left\{a_{j}\right\}_{j \geq 0}$ a sequence of complex numbers such that $\left|a_{j}\right| \leq 1$. Let $\mu$ be the Riesz product $\prod_{j \geq 0}\left[1+\operatorname{Re}\left(a_{j} e^{i \lambda_{j} x}\right)\right]$, that is, the weak limit of measures on $T$ the density of which are the partial products. Then if $\sum_{j \geq 0}\left|\alpha_{j}\right|^{2}<\infty$, the series $\sum_{j \geq 0} \alpha_{j}\left(e^{i \lambda_{j} x}-\frac{1}{2} \bar{a}_{j}\right)$ converges for $\mu$-almost every $x$. The $\mu$-a.e. convergence of series $\sum \alpha_{j} e^{i n \lambda_{j} x}$ is also investigated as well as the case of Riesz products on a compact commutative group.

1980 Mathematics subject classification (Amer. Math. Soc.) (1985 Revision): 42 A 55, 42 A 61, 43 A 25.
\end{abstract}

\section{Introduction}

Let $\Lambda=\left\{\lambda_{j}\right\}_{j \geq 0}$ be a sequence of positive integers such that $\lambda_{j+1} / \lambda_{j} \geq 3$ for all $j$, unless otherwise stated. If $a=\left\{\alpha_{j}\right\}_{j \geq 0}$ is a sequence of complex numbers of moduli less than or equal to 1 , we denote by $\mu_{a}$ the Riesz product $\prod_{j \geq 0}\left(1+\operatorname{Re} a_{j} e^{i \lambda_{j} x}\right)$, that is, the measure which is the weak limit of partial products.

We know that the family $\left\{e^{i \lambda_{j} x}-\frac{1}{2} \bar{a}_{j}\right\}$ of functions on $\mathbf{T}=\mathbb{R} / 2 \pi \mathbb{Z}$ is an orthogonal system in $L^{2}\left(\mu_{a}\right)$ and that the series $\sum \alpha_{j}\left(e^{i \lambda_{j} x}-\frac{1}{2} \bar{a}_{j}\right)$ converges in $L^{2}\left(\mu_{a}\right)$ provided that $\sum\left|\alpha_{j}\right|^{2}<\infty$. It is therefore natural to inquire

(C) 1990 Australian Mathematical Society $0263-6115 / 90 \$ A 2.00+0.00$ 
about the $\mu_{a}$-almost everywhere convergence of such a series. The present paper answers this question and its main result is the following.

ThEOREM. There exists a constant $C$ such that, for any $\Lambda=\left\{\lambda_{j}\right\}_{j \geq 0}$ with $\lambda_{j+1} / \lambda_{j} \geq 3$ and for any $a=\left\{a_{j}\right\}_{j \geq 0}$ with sup $\left|a_{j}\right| \leq 1$, we have

$$
\int \sup _{n \geq 0}\left|\sum_{j=0}^{n} \alpha_{j}\left(e^{i \lambda_{j} x}-\frac{1}{2} \bar{a}_{j}\right)\right|^{2} d \mu_{a}(x) \leq C \sum_{j \geq 0}\left|\alpha_{j}\right|^{2}
$$

for any sequence $\left\{\alpha_{j}\right\}_{j \geq 0}$ of complex numbers.

Such a problem has already been investigated: in [3], the almost everywhere convergence is proved under the hypotheses $\lambda_{j+1} / \lambda_{j} \geq 5$ and $\limsup _{j \rightarrow \infty}\left|a_{j}\right|<\frac{1}{2}$.

The following section is devoted to proving this result. In the last one we deal with series $\sum_{j \geq 0} \alpha_{j}\left(e^{i n \lambda_{j} x}-\hat{\mu}_{a}\left(-n \lambda_{j}\right)\right)$ and briefly discuss the case of Riesz products on arbitrary compact commutative groups.

\section{Proof of the main result}

The proof of the above theorem relies on results of [3]: Lemma 1, Proposition 1 and partly Proposition 2 are from [3], but, for the reader's convenience, these results have been restated and sketches of proof given.

We begin with two lemmas.

LeMma 1. Let $\mu$ and $\nu$ be two positive bounded Borel measures on $\mathbf{T}$. Then the formula $(f \mu) * \nu=(T f) \mu * \nu$ defines a bounded linear map from $L^{1}(\mu)$ to $L^{1}(\mu * \nu)$. Moreover

(1) for any $p \in[1,+\infty],\|T f\|_{L^{p}(\mu * \nu)} \leq\|\nu\|^{1 / p}\|f\|_{L^{p}(\mu)}$, and

(2) $\left\|\sup _{n}\left|T f_{n}\right|\right\|_{L^{p}(\mu * \nu)} \leq\|\nu\|^{1 / p}\left\|\sup \left|f_{n}\right|\right\|_{L^{p}(\mu)}$.

Proof. Assertion (1) is obtained by interpolating between $L^{1}$ and $L^{\infty}$. The second one follows then from the fact that $T$ transforms nonnegative functions into nonnegative ones.

Lemma 2. Let $\mu$ and $\nu$ be two positive bounded Borel measures on T. We then have

(1) $\left\|(\check{g} * \nu)^{\vee}\right\|_{L^{p}(\mu)} \leq\|\nu\|^{1-1 / p}\|g\|_{L^{p}(\mu * \nu)}$ for any $p \in[1,+\infty]$ and for any $g \in L^{1}(\mu * \nu)$ (where $\check{g}(x)=g(-x)$ ), and 
(2) $\left\|\sup _{n}\left|\left(\check{g}_{n} * \nu\right)^{\vee}\right|\right\|_{L^{p}(\mu)} \leq\|\nu\|^{1-1 / p}\left\|\sup _{n} \mid g_{n}\right\| \|_{L^{p}(\mu * \nu)}$.

Proof. The mapping $g \mapsto(\check{g} * \nu)^{\vee}$ is easily checked to be the transpose of $T$. This proves assertion (1) for $1<p \leq \infty$. For $p=1$, it can be checked directly. Assertion (2) follows from the positivity of the map $g \mapsto(\check{g} * \nu)^{\vee}$.

The following two propositions deal with the case $\lambda_{j+1} / \lambda_{j} \geq 5$ and $\sup \left|a_{j}\right|$ $\leq 1 / 4$.

Proposition 1. There exists a constant $K$ such that if $\lambda_{j+1} / \lambda_{j} \geq 5$ and $\sup \left|a_{j}\right| \leq 1 / 4$, we have

$$
\begin{gathered}
\int \sup _{n>0}\left|\prod_{0 \leq j<n}\left(1+\frac{\operatorname{Re}\left(b_{j} e^{i \lambda_{j} x}+c_{j} e^{2 i \lambda_{j} x}\right)}{1+\operatorname{Re}\left(a_{j} e^{i \lambda_{j} x}\right)}\right)-1\right|^{2} d \mu_{a}(x) \\
\quad \leq 4\left[\prod_{j \geq 0}\left(1+K\left(\left|b_{j}\right|^{2}+\left|c_{j}\right|^{2}\right)\right)-1\right]
\end{gathered}
$$

for any sequences $\left\{b_{j}\right\}$ and $\left\{c_{j}\right\}$ of complex numbers.

SKeTCH OF THE PROOF. Let $\left\{\omega_{j}\right\}_{j \geq 0}$ be a sequence of independent Bernoulli random variables such that $P\left(\omega_{j}=1\right)=P\left(\omega_{j}=-1\right)=\frac{1}{2}$. Let $\sigma$ be a number between $\frac{1}{2}$ and 1 . Set

$$
\begin{aligned}
\mu & =\prod_{j \geq 0}\left(1+2 \sigma^{-1} \omega_{2 j} \operatorname{Re}\left(a_{j} e^{i \lambda_{j} x}\right)\right), \\
\nu & =\prod_{j \geq 0}\left(1+\sigma \omega_{2 j} \cos \lambda_{j} x+(1-\sigma) \omega_{2 j+1} \cos 2 \lambda_{j} x\right), \\
f_{n}(x) & =\prod_{0 \leq j<n}\left[1+\frac{2 \operatorname{Re}\left(\sigma^{-1} \omega_{2 j} b_{j} e^{i \lambda_{j} x}+(1-\sigma)^{-1} \omega_{2 j+1} c_{j} e^{2 i \lambda_{j} x}\right)}{1+2 \sigma^{-1} \omega_{2 j} \operatorname{Re}\left(a_{j} e^{i \lambda_{j} x}\right)}\right]-1
\end{aligned}
$$

(the hypothesis $\lambda_{j+1} / \lambda_{j} \geq 5$ ensures the existence of the measure $\nu$ ). We have $\mu * \nu=\mu_{a}$ and

$$
T f_{n}(x)=\prod_{0 \leq j<n}\left[1+\frac{\operatorname{Re}\left(b_{j} e^{i \lambda_{j} x}+c_{j} e^{2 i \lambda_{j} x}\right)}{1+\operatorname{Re}\left(a_{j} e^{i \lambda_{j} x}\right)}\right]-1
$$

(where $T$ is the operator described in Lemma 1).

Then apply Lemma 1, part (2), take the expectations of both sides, use Doob's inequality on $L^{2}$-bounded martingales and majorize suitably the right hand side. As previously said, detailed calculations can be found in [3, pages 146-151]. The value of $K$ which can be obtained that way is $16 \sigma^{2}(1-\sigma)^{-2}\left(4 \sigma^{2}-1\right)^{-1}$. 
LEMMA 3. If $\left\{x_{j}\right\}$ is a finite sequence of nonnegative numbers the sum of which is less than 1 , we have

$$
\prod\left(1+x_{j}\right)-1 \leq\left(\sum x_{j}\right) /\left(1-\sum x_{j}\right) .
$$

Proof. We clearly have $\Pi\left(1+x_{j}\right) \leq 1+\sum x_{j}+\sum x_{j} x_{k}+\sum x_{j} x_{k} x_{l}+\cdots$.

LEMMA 4. Let $\left\{x_{j}\right\}_{j \geq 0}$ be a sequence of complex numbers. We have

$$
\sup _{n>0}\left|\sum_{0 \leq j<n} x_{j}\right| \leq \lim _{\tau \rightarrow 0} \inf |\tau|^{-1} \sup _{n>0}\left|\prod_{0 \leq j<n}\left(1+\tau x_{j}\right)-1\right| .
$$

Proof. We have

and therefore

$$
\sum_{0 \leq j<n} x_{j}=\lim _{\tau \rightarrow 0} \tau^{-1}\left(\prod_{0 \leq j<n}\left(1+\tau x_{j}\right)-1\right),
$$

$$
\begin{aligned}
\left|\sum_{0 \leq j<n} x_{j}\right| & =\lim _{\tau \rightarrow 0}|\tau|^{-1}\left|\prod_{0 \leq j<n}\left(1+\tau x_{j}\right)-1\right| \\
& \leq \liminf _{\tau \rightarrow 0} \sup _{n \geq 0}|\tau|^{-1}\left|\prod_{0 \leq j<n}\left(1+\tau x_{j}\right)-1\right| .
\end{aligned}
$$

Proposition 2. If $\lambda_{j+1} / \lambda_{j} \geq 5$ and $\sup \left|a_{j}\right| \leq 1 / 4$, we have

$$
\int \sup _{n>0}\left|\sum_{0 \leq j<n} \alpha_{j}\left(e^{i \lambda_{j} x}-\frac{1}{2} \bar{a}_{j}\right)\right|^{2} d \mu_{a}(x) \leq 128 K \sum_{j \geq 0}\left|\alpha_{j}\right|^{2}
$$

for any sequence $\left\{\alpha_{j}\right\}$ of complex numbers.

This proposition improves [3, Corollary 3.4].

Proof. The following formulas can be checked:

$$
\begin{aligned}
& \cos \lambda_{j} x-\frac{1}{2} \operatorname{Re} a_{j}=\frac{\operatorname{Re}\left[\left(1-\frac{1}{4}\left|a_{j}\right|^{2}-\frac{1}{4} a_{j}^{2}\right) e^{i \lambda_{j} x}+\frac{1}{2} a_{j} e^{2 i \lambda_{j} x}\right]}{1+\operatorname{Re}\left(a_{j} e^{i \lambda_{j} x}\right)} ; \\
& \sin \lambda_{j} x+\frac{1}{2} \operatorname{Im} a_{j}=\frac{-\operatorname{Re}\left[i\left(1-\frac{1}{4}\left|a_{j}\right|^{2}+\frac{1}{4} a_{j}^{2}\right) e^{i \lambda_{j} x}+\frac{1}{2} i a_{j} e^{2 i \lambda_{j} x}\right]}{1+\operatorname{Re}\left(a_{j} e^{i \lambda_{j} x}\right)} .
\end{aligned}
$$

Let $\left\{u_{j}(x)\right\}_{j \geq 0}$ be either one of the sequences $\left\{\cos \lambda_{j} x-\frac{1}{2} \operatorname{Re} a_{j}\right\}$ or $\left\{\sin \lambda_{j} x+\frac{1}{2} \operatorname{Im} a_{j}\right\},\left\{\alpha_{j}\right\}_{j \geq 0}$ an arbitrary sequence of real numbers such 
that $\sum_{j \geq 0} \alpha_{j}^{2}<\infty$ and $\left\{\tau_{k}\right\}_{k \geq 0}$ a sequence of positive numbers converging towards 0 . The above formulas and Proposition 1 give

$$
\int \sup _{n>0}\left|\prod_{0 \leq j<n}\left(1+\tau_{k} \alpha_{j} u_{j}\right)-1\right|^{2} d \mu_{a} \leq 4\left[\prod_{j \geq 0}\left(1+2 K \tau_{k}^{2} \alpha_{j}^{2}\right)-1\right] .
$$

Using Lemmas 3 and 4 we get

$$
\begin{aligned}
\int \sup _{n>0}\left|\sum_{0 \leq j<n} \alpha_{j} u_{j}\right|^{2} d \mu_{a} & \leq \int \liminf _{k \rightarrow \infty} \sup _{n>0} \tau_{k}^{-1}\left|\prod_{0 \leq j<n}\left(1+\tau_{k} \alpha_{j} u_{j}\right)-1\right|^{2} d \mu_{a} \\
& \leq \liminf _{k \rightarrow \infty} \int \sup _{n>0} \tau_{k}^{-2}\left|\prod_{0 \leq j<n}\left(1+\tau_{k} \alpha_{j} u_{j}\right)-1\right|^{2} d \mu_{a} \\
& \leq \liminf _{k \rightarrow \infty} 4 \tau_{k}^{-2}\left[\prod_{0 \leq j<n}\left(1+2 K \tau_{k}^{2} \alpha_{j}^{2}\right)-1\right] \\
& \leq 8 K \sum_{j \geq 0} \alpha_{j}^{2} .
\end{aligned}
$$

Now, adding the estimates corresponding to both choices of $u_{j}$, we get

$$
\int \sup _{n>0}\left|\sum_{0 \leq j<n} \alpha_{j}\left(e^{i \lambda_{j} x}-\frac{1}{2} \bar{a}_{j}\right)\right|^{2} d \mu_{a}(x) \leq 32 K \sum_{j \geq 0} \alpha_{j}^{2} .
$$

We wish now to remove the restrictions on $\lambda_{j}$ 's as well as on $a_{j}$ 's. Let us denote by $\mathbb{P}(\kappa, \tau)$ the following property: there exists a constant $M$ such that, if $\inf \lambda_{j+1} / \lambda_{j} \geq \kappa$ and $\sup \left|a_{j}\right| \leq \tau$, we have

$$
\int \sup _{n>0}\left|\sum_{0 \leq j<n} \alpha_{j}\left(e^{i \lambda_{j} x}-\frac{1}{2} \bar{a}_{j}\right)\right|^{2} d \mu_{a}(x) \leq M \sum_{j \geq 0}\left|\alpha_{j}\right|^{2}
$$

for any sequence $\left\{\alpha_{j}\right\}$ of complex numbers.

Proposition 2 exactly says that $\mathbb{P}(5,1 / 4)$ holds and we have to prove that $\mathbb{P}(3,1)$ holds. This will be done by applying twice the following lemma.

Lemma 5. $\mathbb{P}(5, \kappa)$ implies $\mathbb{P}(3, \inf (2 \kappa, 1))$.

Proof. We suppose $\mathbb{P}(5, \kappa)$ is true with constant $M$. Let $\Lambda=\left\{\lambda_{j}\right\}_{j \geq 0}$ 
and $\left\{a_{j}\right\}_{j \geq 0}$ be such that $\lambda_{j+1} / \lambda_{j} \geq 3$ and $\left|a_{j}\right| \leq \inf (2 \kappa, 1)$. Let us set

$$
\begin{gathered}
\mu=\mu_{a}, \quad \nu=\prod_{j \geq 0}\left(1+\cos \lambda_{2 j} x\right), \\
g_{n}(x)=\sum_{0 \leq j<n} \alpha_{2 j}\left(e^{i \lambda_{2 j} x}-\frac{1}{4} \bar{a}_{2 j}\right) .
\end{gathered}
$$

We then have

$$
\mu * \nu=\prod_{j \geq 0}\left(1+\frac{1}{2} \operatorname{Re}\left(a_{2 j} e^{i \lambda_{2 j} x}\right)\right)
$$

and

$$
\left(\check{g}_{n} * \nu\right)^{\vee}(x)=\frac{1}{2} \sum_{0 \leq j<n} \alpha_{2 j}\left(e^{i \lambda_{2 j} x}-\frac{1}{2} \bar{a}_{2 j}\right) .
$$

So, by Lemma 2 , part (2), and $\mathbb{P}(5, \kappa)$ we obtain

$$
\begin{aligned}
& \int \sup _{n>0}\left|\sum_{0 \leq j<n} \alpha_{2 j}\left(e^{i \lambda_{2 j} x}-\frac{1}{2} \bar{a}_{2 j}\right)\right|^{2} d \mu_{a}(x) \\
& \quad \leq 4 \int \sup _{n>0}\left|\sum_{0 \leq j<n} \alpha_{2 j}\left(e^{i \lambda_{2 j} x}-\frac{1}{4} \bar{a}_{2 j}\right)\right|^{2} d \mu * \nu(x) \\
& \quad \leq 4 M \sum_{j \geq 0}\left|\alpha_{2 j}\right|^{2} .
\end{aligned}
$$

The odd terms are taken care of similarly. Eventually we get

$$
\int \sup _{n>0}\left|\sum_{0 \leq j<n} \alpha_{j}\left(e^{i \lambda_{j} x}-\frac{1}{2} \bar{a}_{j}\right)\right|^{2} d \mu_{a}(x) \leq 8 M \sum_{j \geq 0}\left|\alpha_{j}\right|^{2} .
$$

\section{Further results and remarks}

First we investigate the convergence of series of the form

$$
\sum_{j \geq 0} \alpha_{j}\left[e^{i k \lambda_{j} x}-\hat{\mu}\left(-k \lambda_{j}\right)\right]
$$

Proposition 3. There exists a constant $C$ such that, for any integer $k \geq 2$, and for any sequences $\left\{\lambda_{j}\right\}$ and $\left\{a_{j}\right\}$ such that $\inf \lambda_{j+1} / \lambda_{j} \geq 2 k+1$ and $\sup \left|a_{j}\right| \leq 1$, we have

$$
\int \sup _{n>0}\left|\sum_{0 \leq j<n} \alpha_{j} e^{i k \lambda_{j} x}\right|^{2} d \mu_{a}(x) \leq C \sum_{j \geq 0}\left|\alpha_{j}\right|^{2}
$$

for any sequence $\left\{\alpha_{j}\right\}$ of complex numbers. 
The proof of this proposition follows exactly the same lines as the one of the main theorem; Propositions 1 and 2 and Lemma 5 have to be revisited.

COROLlary. There exists a constant $C$ such that, for any sequences $\left\{\lambda_{j}\right\}$ and $\left\{a_{j}\right\}$ such that $\inf \lambda_{j+1} / \lambda_{j} \geq 3, \lim _{j \rightarrow \infty} \lambda_{j+1} / \lambda_{j}=+\infty$ and $\sup \left|a_{j}\right| \leq 1$, we have

$$
\int \sup _{n>0}\left|\sum_{0 \leq j<n} \alpha_{j}\left[e^{i k \lambda_{j} x}-\hat{\mu}_{a}\left(-k \lambda_{j}\right)\right]\right|^{2} d \mu_{a} \leq C \phi(2 k+1) \sum_{j \geq 0}\left|\alpha_{j}\right|^{2}
$$

where

$$
\phi(t)=\inf \left\{j \geq 1 ; \lambda_{n} / \lambda_{n-1} \geq t \text { for all } n \geq j\right\} .
$$

This corollary, in the case where $\phi(2 k+1)$ and $\phi\left(k+\frac{3}{2}\right)$ are comparable, leads to a slight improvement of [3, Proposition 2.7]. But more useful an improvement of this proposition is the one by Brown, Moran and Pearce [1]: they allow $\left\{\lambda_{j}\right\}$ to satisfy a mixture of divisibility and lacunarity properties and this enables them to obtain the Hausdorff dimension of sets of numbers which are normal with respect to some bases and abnormal with respect to some others.

Now, let us briefly examine how this works in the general group setting. Let $G$ be a compact commutative group and $\Gamma$ its dual. As in [2], a subset $\Lambda$ of $\Gamma$ is said to be dissociate if any $\gamma \in \Gamma$ has at most one representation of the form $\gamma=\sum_{\lambda \in \Lambda} \varepsilon_{\lambda} \lambda$, where $\varepsilon_{\lambda} \in\{0,1\}$ if $2 \lambda=0, \varepsilon_{\lambda} \in\{-1,0,1\}$ otherwise and all but a finite number of the $\varepsilon_{\lambda}$ 's are zero. Similarly $\Gamma$ is 2dissociate if any $\gamma \in \Gamma$ has at most one representation of the form $\gamma=\sum \varepsilon_{\lambda} \lambda$, where $\varepsilon_{\lambda} \in\{0,1\}$ if $2 \lambda=0, \varepsilon_{\lambda} \in\{ \pm 1,0\}$ if $3 \lambda=0, \varepsilon_{\lambda} \in\{0, \pm 1,2\}$ if $4 \lambda=0, \varepsilon_{\lambda} \in\{0, \pm 1, \pm 2\}$ otherwise and all but a finite number of the $\varepsilon_{\lambda}$ 's are zero. In that setting the counterpart of the main result is the following

THEOREM. There exists a constant $C$ such that if $\Lambda$ is a countable dissociate set which is the union of $N$ 2-dissociate sets, then for any Riesz product constructed over $\Gamma$, and for any enumeration $\lambda_{0}, \lambda_{1}, \ldots, \lambda_{n}, \ldots$ of $\Lambda$, we have

$$
\int \sup _{n>0}\left|\sum_{0 \leq j<n} \alpha_{j}\left[\left(\lambda_{j}, x\right\rangle-\hat{\mu}\left(-\lambda_{j}\right)\right]\right|^{2} d \mu(x) \leq N C \sum_{j \geq 0}\left|\alpha_{j}\right|^{2} .
$$

Therefore, in the case of Hadamard sets, although for their definition the ordering is important, the series $\sum \alpha_{j}\left(e^{i \lambda_{j} x}-\frac{1}{2} \bar{a}_{j}\right)$ converges $\mu_{a}$-almost everywhere whatever the ordering of terms may be. 


\section{Acknowledgement}

This work was done while the author was enjoying the hospitality of the University of New South Wales.

\section{References}

[1] G. Brown, W. Moran and C. E. M. Pearce, 'Riesz products, Hausdorff dimension and normal numbers.' Math. Proc. Cambridge Philos. Soc. 101 (1987), 529-540.

[2] E. Hewitt and H. S. Zuckerman, 'Singular measures with absolutely continuous squares,' Proc. Cambridge Philos. Soc. 62 (1966), 399-420. Corrigendum ibid. 63 (1967), 367368.

[3] J. Peyrière, 'Etude de quelques propriêtés des produits de Riesz,' Ann. Inst. Fourier 25 (1975), 127-169.

Université de Paris-Sud

Unité Associée au CNRS no 757

Mathématiques, bât. 425

91405 Orsay Cedex

France 